\title{
A INFLUÊNCIA DA MASSAGEM CLÁSSICA CORPORAL RELAXANTE ASSOCIADA À AROMATERAPIA NA QUALIDADE DO SONO E ESTRESSE EM PROFESSORAS UNIVERSITÁRIAS.
}

\author{
Elba Karine Souza Pereira, Elisa Araújo de Santana, Ester Braga Cruz da Silva, \\ Ironaide Ferreira do Nascimento, Felipe Scholz Ramos e André Leonardo da Silva
}

Nessi

Escola da Ciências da Saúde , Universidade Anhembi Morumbi - UAM

Email: elisaarsantana@gmail.com

\section{RESUMO}

Introdução: Segundo a Associação Brasileira de Medicina do Sono as atividades dos dias atuais têm dificultado a qualidade e tempo de sono, causando danos ao Sistema Nervoso Central e liberando substâncias diretamente relacionadas ao estresse, assim as alterações do metabolismo na falta de algumas horas de sono por noite equiparam-se ao de um idoso. Objetivo: Verificar a influência da massagem clássica corporal (relaxante) associada à aromaterapia na qualidade do sono e estresse em professoras universitárias. Metodologia: Foi realizado um estudo exploratório e qualitativo, com amostra de 14 professoras universitárias, através de questionários e práticas de massagem com associação dos óleos essenciais de Olíbano e Cedro, controlado os resultados com oxímetro e medidor de pressão digital. Resultados: Embora não tenha dado diferença estatística vale ressaltar que o óleo de cedro reduziu de maneira considerada a Frequência cardíaca das voluntárias. Todas as voluntárias relataram haver melhora na qualidade do sono referente a noite do dia de recebimento da massagem, como a diminuição das dores, sono constante durante a noite, levando-nos a confirmar o grande efeito que a massagem relaxante exerce no indivíduo. Conclusão: Embora os resultados da inalação dos óleos essenciais não tenham dado diferenças estatísticas, podemos concluir que esse procedimento é eficaz na melhora do estresse e gradativamente na melhora da qualidade do sono.

Palavras-chave: Massagem, sono, estresse, professores universitários.

\section{ABSTRACT}

Introduction: According to the Brazilian Association of Sleep Medicine, the activities of the present day have hampered the quality and time of sleep, causing damage to the Central Nervous System and releasing substances directly related to stress, thus the metabolism changes in the absence of a few hours of sleep by night they were equated with that of an old man. Objective: To verify the influence of classic (relaxing) body massage associated with atherapy in sleep quality and stress in Fórum Internacional de Qualidade de Vida e Saúde - Curitiba, 15 de junho de 2019 
university teachers. Methodology: An exploratory and qualitative study was carried out, with a sample of 14 university teachers, through questionnaires and massage practices with association of the essential oils of Olíbano and Cedro, controlled the results with oximeter and Power Lab. Results: Although there was no difference it is worth noting that cedar oil reduced in a manner considered the Heart Rate of the volunteers. All volunteers reported that there was improvement in sleep quality regarding the night of the day of receiving the massage, such as decreased pain, constant sleep at night, leading us to confirm the great effect that relaxing massage exerts on the individual. Conclusion: Although the inhalation results of essential oils have not given statistical differences, we can conclude that this procedure is effective in improving stress and gradually improving the quality of sleep.

Keywords: Massage, sleep, stress, university professors.

\section{INTRODUÇÃO}

Segundo a Organização Mundial da Saúde, o estresse está gradativamente presente na vida do ser humano sendo na atualidade uma das principais causas de mortes entre 7 e 10 pessoas em países desenvolvidos. (Barros, 2017).

Estudos mostram que o estresse causa apneia, insônia, doenças, transtornos psicológicos entre outras disfunções, pode ser considerado a doença do século $\mathrm{XX}$, sendo uma reação do organismo contra as reações internas ou externas que se excessivas e contínuas podem causar doenças, desconfortos, irritações, problemas psicológicos, frustações, medos, entre outros problemas emocionais (Barros, 2017). Essas reações podem ser expressadas em três fases: do alarme, da resistência e de esgotamento (Zular, 2000).

Diferentemente do estresse em que o corpo está com o sistema simpático ativo, o sono é caracterizado por um desligamento desse sistema responsável pela "luta ou fuga", ou seja, o sistema parassimpático é ativado (Bertolazi, 2008). Este desligamento faz com que o corpo não responda mais aos estímulos externos, porém quando o sono se torna de má qualidade, ocorrem diferentes problemas que refletem em situações diárias como no trabalho, nos estudos ou em executar uma simples tarefa como a leitura, podendo ocasionar em acidentes de carro e outros equivalentes a esse. Portanto, esses aspectos influenciam diretamente no bem-estar e vida do indivíduo sendo o motivo para a procura de ajuda médica e tendo como tratamento complementar a massagem relaxante.

A massagem relaxante tem efeito fisiológico nas terminações nervosas que existem na pele, contribuindo na melhora da vasodilatação, no auxílio da eliminação 
de resíduos extracelulares, melhora a circulação arterial e venosa, aumento na produção de endorfinas, tem efeito sedativo e analgésico, aumento na atuação do sistema linfático e relaxamento muscular, podendo assim ter efeitos reflexos ou mecânicos (Perez, 2014).

Assim sendo, essa terapia auxilia na diminuição do estresse, melhorando o rendimento dos professores que são tão importantes para a vida acadêmica de seus alunos, refletindo diretamente no número exacerbado de procura médica na melhora da qualidade de vida. De acordo com essas informações, a pesquisa teve como propósito verificar a influência da massagem relaxante corporal associada a aromaterapia em professores universitários na melhora da qualidade do sono e diminuição do estresse.

A massagem clássica corporal relaxante é constituída por 29 manobras sendo elas: Deslizamento profundo, amassamento, fricção, vibração e percussão. Através de movimentos realizados com as mãos sobre o corpo humano com o objetivo de atuar em diversas áreas e finalidades diferentes seguindo uma ordem específica. Massagem é o ato de tocar com as mãos provocando alguma reação, seja ela psicológica, química ou mecânica. (Nessi, 2010).

Assim como a massagem clássica relaxante, a aromaterapia tem muitos benefícios. Realizada através de técnicas específicas, atua nas áreas física ou emocional. Isso ocorre através do olfato quando o aroma é inalado causando emoções e sentimentos nos seres humanos, resultando no bem-estar. (Pereira, 2013)

Atualmente utilizada em clínicas de estética e SPAs, como complemento aos tratamentos holísticos. Quando o indivíduo é exposto a aromas diferentes, o sistema nervoso é acionado gerando respostas físicas e psicológicas. O que define os bons óleos essenciais ricamente aromatizados extraídos de flores, sementes, folhas e cascas são: a forma e o cuidado em que essas fontes foram tratadas e extraídas. Sendo muito utilizadas em escalda-pés, sauna, hidromassagem, ofurô, massagem terapêutica e compressas (Fernandes, Maxwell, 2008).

O óleo essencial utilizado na aromaterapia, diferente dos óleos utilizados em cozinha, é de baixa viscosidade, parecido com o éter ou álcool. É um produto muito 
utilizado na estética, em cosméticos e medicamentos, sendo concentrado e instável. Tem o cheiro característico da planta de origem e possui preço elevado por necessitar de uma quantidade grande de plantas para a extração do óleo. Eles possuem propriedades medicinais, auxiliando em todos os sistemas do corpo, por serem compatíveis com a pele, musculatura, circulação e respiração exceto a mucosa visual (Laszlo, 2013). Desta forma podemos visualizar diferentes plantas que podem ser utilizadas para objetivos terapêuticos, dentre elas o óleo essencial de Olíbano e Cedro.

Olíbano (Boswellia serrata), pertencente à família Burseraceae, é uma árvore ramificada de tamanho médio a grande porte, encontrada em regiões secas e montanhosas na Índia, no norte da África e no Oriente Médio. A resina obtida da casca (tronco) dessa planta é conhecida por seu papel importante na medicina Ayurveda e mais recentemente tem sido utilizada pela sua atuação em processos inflamatórios. (Naturell, 2016). O óleo essencial Boswellia serrata é aprovada pelo FDA e pelo Conselho Europeu também para uso alimentício, suas propriedades terapêuticas e medicinais são anti-inflamatório, adstringente, coagulante (ferimentos) e vasodilatador usados para a tensão. (Paganini, Silva, 2014)

Já o óleo essencial de cedro (Juníperus virginiana) pertence à família Crupressaceae, é uma planta cultivada e tem origem na América do Norte. É uma árvore conífera com $+/-12 \mathrm{~m}$, com frutos azulados quando imaturos, a parte da planta usada são as folhas e seus principais componentes são limoneno, cedreno e cedrol (Santos 1998). Suas propriedades terapêuticas e medicinais são: sedativa, relaxante, adstringente, diurético, expectorante, repelente de insetos usados para ansiedade e estresse. (Paganini, silva, 2013).

Os óleos essenciais, quando utilizados por um profissional com conhecimento e aplicados com métodos e dosagens corretas permitem o equilíbrio do organismo nas dimensões físicas, mentais e espirituais, sempre é valido lembrar que para os efeitos desejados são necessários à utilização de óleos essenciais com qualidade. (Paganini, Silva, 2014). Na massagem relaxante os óleos essenciais podem ser diluídos em óleos vegetais ou cremes neutros pois atuarão como carreadores.

O óleo vegetal semente de uva é bastante conhecido por sua associação aos óleos essenciais nas terapias. Extraído da planta denominada Vitis vinífera, muito Fórum Internacional de Qualidade de Vida e Saúde - Curitiba, 15 de junho de 2019 
produzido na Europa, em comparação, no Brasil sendo ainda pouco conhecido. Ele cresce em regiões em que a incidência de sol é alta para se obter a qualidade do mesmo. Com o aumento da produção de uvas, aumentou-se os resíduos de biomassa, e com isso verificou-se a importância dessa reutilização principalmente pelo conteúdo ser rico em lipídeos e antioxidantes como a vitamina $E$, sendo que suas concentrações podem variar dependendo da forma em que ele foi processado. Agindo através da hidratação e contra o envelhecimento cutâneo é um dos óleos mais indicados e utilizados para esses fins (Shinagawa, 2015).

O Objetivo desta pesquisa é verificar a influência da massagem clássica corporal (relaxante) associada à aromaterapia na qualidade do sono e estresse em professoras universitárias.

\section{METODOLOGIA}

Essa é uma pesquisa de delineamento experimental, onde, catorze professoras voluntárias da Universidade Anhembi Morumbi com a faixa etária entre 28 a 50 anos, receberão a técnica de massagem clássica corporal (relaxante) associada aos óleos essenciais de Cedro (Juniperus Virginiana) e Olíbano (Boswellia serrata). Os óleos essenciais utilizados foram da empresa doTERRA.

A intervenção experimental foi dada somente após aprovação do comitê de ética e após todas as voluntárias assinarem o TCLE (Termo de conscientização livre e esclarecido) (Apêndice I).

O procedimento foi padronizado, iniciando através do preenchimento das fichas, seguido da massagem clássica corporal relaxante, com a quantidade de 29 manobras, iniciando em decúbito dorsal, seguindo a ordem através dos membros inferiores, braços, mãos, abdômen, colo, ombros e trapézio, e finalizando em decúbito frontal, seguindo a ordem através dos membros inferiores, em seguida dorso e cabeça. Com duração de quarenta minutos, em uma única sessão, no laboratório da Universidade Anhembi Morumbi, campus Mooca-SP. O público foi dividido em dois grupos conforme quadro 1. 
Quadro 1 - Divisão dos Grupos

\begin{tabular}{|l|l|l|}
\hline GRUPO (G) & OBJETIVO & Qtde de Pessoas \\
\hline $\begin{array}{l}\text { Grupo } \\
\text { (G1) }\end{array}$ & $\begin{array}{l}\text { Massagem + inalação do óleo essencial de } \\
\text { Olíbano }\end{array}$ & № = 7 pessoas \\
\hline $\begin{array}{l}\text { Grupo } \\
\text { (G2) }\end{array}$ & $\begin{array}{l}\text { Massagem + inalação do óleo essencial de } \\
\text { Cedro }\end{array}$ & № = 7 pessoas \\
\hline
\end{tabular}

Para coleta dos resultados foi solicitado prévia de trinta minutos em jejum as voluntárias, a fim de se evitar possíveis desconfortos ou alterações de resultados. Em complemento e inicialmente, todas preencheram a ficha de anamnese (Anexo A), questionário de qualidade do sono (Anexo B) e questionário sobre o grau de estresse (Anexo $\mathrm{C}$ ). Os questionários de sono e estresse foram enviados através do endereço de e-mail para as professoras participantes e foram preenchidos no dia seguinte pelas mesmas. Dessa forma, foi avaliado a qualidade de sono e estresse após a técnica.

A pressão arterial e sinais vitais, como: batimentos cardíacos e oxigenação foi avaliado antes e depois da aplicação da massagem e após a inalação do óleo essencial com a cliente sentada, através do monitor de pressão digital verificado no braço esquerdo da G-TECH, e oxímetro de dedo monitor de frequência cardíaca verificado no dedo indicador direito, Fingertip Pulse Oximeter da ChoiceMMed.

Em cada sessão foi realizada a Massagem Clássica Corporal relaxante, e ao término, foi entregue para a professora participante um recipiente fechado com algodão, contendo uma gota do óleo essencial. Sendo, Olibano ou Cedro. Será inalado três vezes consecutivas. Todas as massagens foram realizadas através do óleo vegetal semente de uva para o deslizamento.

\section{ANÁLISE DOS RESULTADOS}

Os resultados da pesquisa foram avaliados através de dados coletados antes, depois da massagem e após a inalação do óleo essencial, foram analisados através de estatísticas e descritos em gráficos a partir de média/ desvio padrão. Os participantes também foram avaliados por meio da escala Pittsburgh (Anexo B), e 
questionário sobre o grau de estresse (Anexo $\mathrm{C}$ ) em dois momentos, no início da técnica e no dia posterior, esses dados foram comparados.

\section{RESULTADOS}

Gráficos 1 e 2 - Média e desvio padrão (pressão arterial) dos batimentos cardíacos dos indivíduos antes de iniciar o procedimento, após o recebimento da massagem e após a inalação dos óleos essenciais de Cedro e Olíbano.

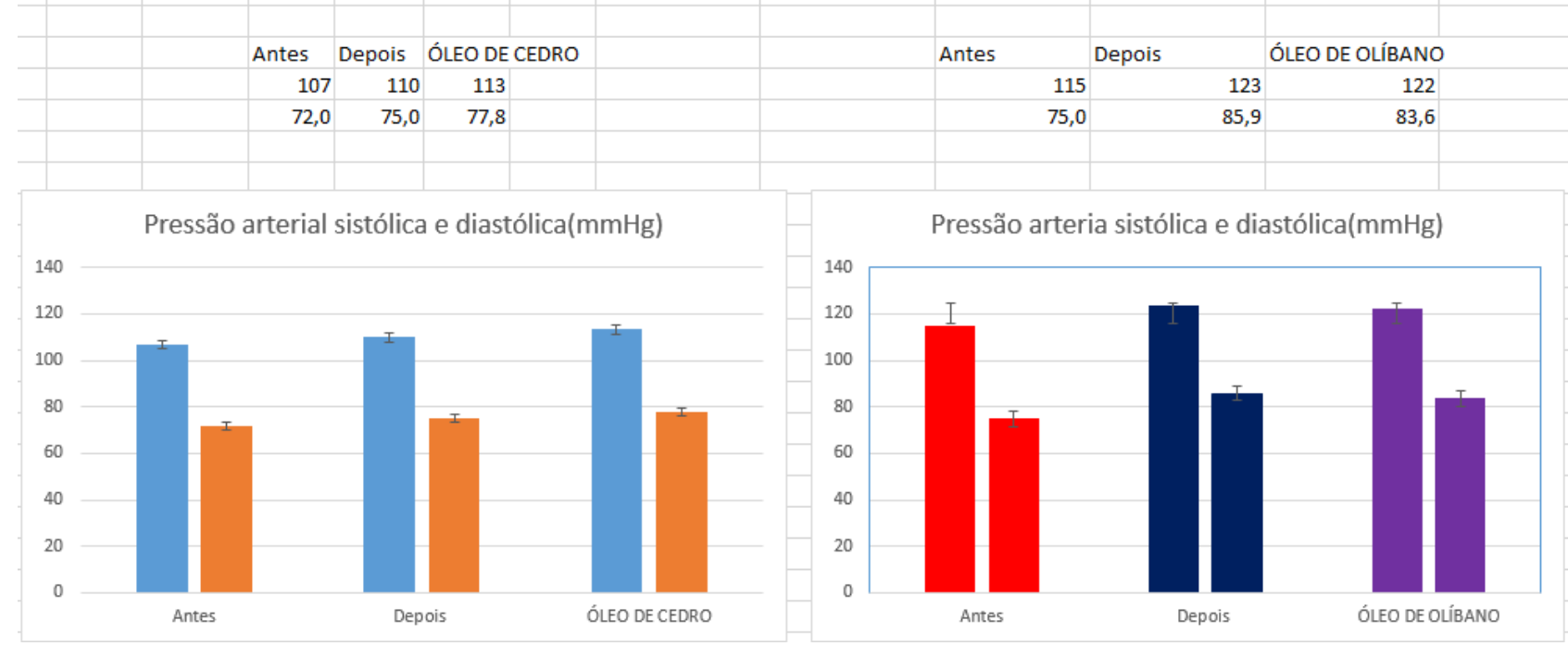

Gráficos 3 e 4- Média e desvio padrão (saturação do Oxigênio e batimentos cardíacos) através do oxímetro antes de iniciar o procedimento, após o recebimento da massagem e após a inalação dos óleos essenciais de Cedro e Olíbano.

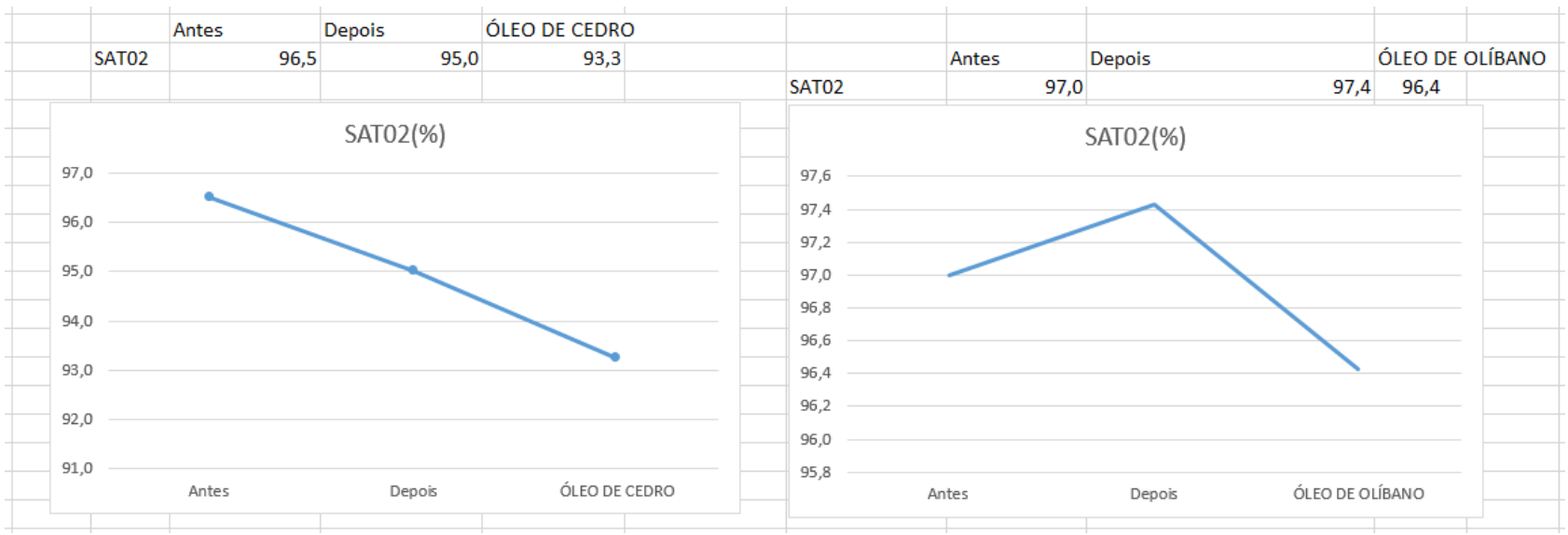


Gráfico 5- Média e desvio padrão (saturação do Oxigênio e batimentos cardíacos) comparação dos óleos essenciais de Cedro e Olíbano, através do oxímetro antes de iniciar o procedimento, após o recebimento da massagem e após a inalação dos óleos essenciais.

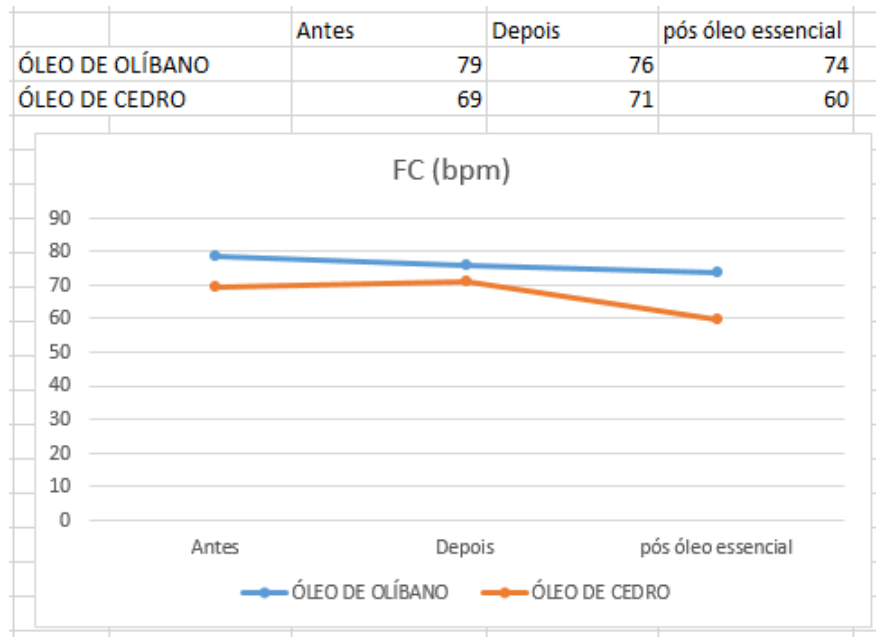

Embora não tenha dado diferença estatística vale ressaltar que o óleo de cedro reduziu de maneira considerada a Frequência cardíaca dos voluntários (Gráfico 5). Há necessidades de maiores pesquisas em maior número para obter resultados conclusivos fisiologicamente.

Através dos questionários realizados antes da prática de massagem relaxante, pode-se perceber que todas as voluntárias têm níveis de estresse alto. As professoras assinalaram em média de 4 a 8 itens no questionário contendo 12 itens no total. Os hábitos mais recorrentes foram: De 14 professoras, 11 relataram sentir tensão muscular, 8 possui esquecimento de coisas corriqueiras, 7 irritabilidade excessiva, 9 ansiedade, 8 distúrbio do sono e 11 relataram cansaço ao levantar. Esses comparativos foram contabilizados através da metade das professoras que assinalaram as mesmas questões.

Em seguida foi preenchido o questionário do sono, sendo a média do horário em que elas dormem entre 23:00hs e 00:30hs, com duração de 4 a 6 horas por noite. Habitualmente demoram em média de 10 a 20 minutos para adormecer, possuindo como horário para despertar entre 5 e 6 horas da manhã. $O$ questionário se refere a qualidade do sono durante a noite, questionando possíveis alterações, 
sendo as mais recorrentes: demorar para adormecer, despertar e levantar durante a noite para ir ao banheiro, sentir frio, calor e ter sonhos ruins. Os pontos mais agravantes apontados no questionário sendo assinalado por 12 de 14 professoras foram: a qualidade do sono no geral classificada como ruim, presença de dor e problemas ao manter-se suficientemente entusiasmado ao realizar as atividades. Foi questionado sobre qual as chances de elas dormirem enquanto fazem atividades diárias, foi apontado de pequena a alta chance de cochilar.

Após a realização da prática de massagem corporal relaxante, associada a inalação dos óleos de cedro e Olíbano pode-se perceber uma melhora considerável do estresse, 5 entre 14 professoras apontaram alto nível de estresse durante o dia seguido do procedimento, enquanto as outras 9 apontaram pouco estresse naquele dia. Todas as voluntárias relataram haver melhora na qualidade do sono referente a noite do dia de recebimento da massagem, como a diminuição das dores, sono constante durante a noite, não constataram frio, calor ou sonhos ruins nesse dia, levando-nos a confirmar o grande efeito que a massagem relaxante exerce no indivíduo.

\section{CONCLUSÃO}

Embora os resultados da inalação dos óleos essenciais não tenham dado diferenças estatísticas, podemos concluir que esse procedimento é eficaz na melhora do estresse e gradativamente na melhora da qualidade do sono. Posteriormente há necessidade de maiores pesquisas em maior número para obter resultados conclusivos fisiologicamente.

\section{REFERÊNCIAS}

AMARAL, F. Técnicas de aplicação de óleos essenciais. São Paulo/ SP, Cengage Learning, 2015.

ANDRADE, L. Sistema de Medição para Oximetria de Pulso. 2009. Dissertação. (Mestrado em Engenharia Biomédica) - Programa de Pós-graduação em Engenharia Elétrica e Informática Industrial, Universidade Tecnológica Federal do Paraná. Curitiba, 2009.

BARROS, D.; FUNKE, G.; LOURENÇO, R. 49 Perguntas sobre estresse. (Coleção 49 perguntas; v. 2). Barueri, SP - Brasil. Manole, 2017. 
BERTOLAZI, A., Tradução, Adaptação Cultural e Validade de dois instrumentos de avaliação do sono: escala de sonolência de Epworth e índice de qualidade de sono de Pittsburgh. Dissertação de Pós-Graduação em Medicina: Ciências Médicas-Universidade Federal do Rio Grande do Sul Faculdade de Medicina, Porto Alegre, 2008.

FERNANDES, M.; Curso didático de estética. (Volume 2). São Caetano do Sul, SP - Brasil. Yends 2008.

GUIRRO, E; GUIRRO, R. Fisioterapia Dermato-Funcional: Fundamentos, Recursos, Patologias. $3^{\circ}$ ed. São Paulo. Manole, 2010.

LIPP, M. Questionário ajuda a identificar nível e sintomas de estresse. Instituto de Psicologia e controle do stress Marilda Emmanuel Novaes Lipp. Disponível em: <https://g1.globo.com/>. Acesso em: 24 Nov. 2018.

MENDES, T. et al. Adequação do uso do oxigênio por meio da oximetria de pulso: um processo importante de segurança do paciente. São Paulo, 2010.

NATURELL. Boswellia Serrata. [S.I.: s.n.], 2016. 4 p. Disponível em: <http://www.naturell.com.br/assets/files/BOSWELLIA-EXTRACT.pdf>. Acesso em: 16 dez. 2018.

NESSI, A. Massagem antiestresse: Uma abordagem Teórica e Prática para o BemEstar. 5ª edição São Paulo: Phorte, 2010.

PAGANINI, T. Bem-estar e qualidade de vida: a aromaterapia no cuidado ao estresse. 2013. 112 p. dissertação (mestrado). (bem estar e qualidade de vida: a aromaterapia no cuidado ao estresse.)- uíniversidade do vale do itajaí, itajaí (sc), 2013. disponível em:

<https://siaiap39.univali.br/repositorio/bitstream/repositorio/1064/1/tatiana\%20pagani ni2013.pdf>. acesso em: 21 dez. 2018.

PAGANINI, T.; FLORES e SILVA, Y. O uso da aromaterapia no combate ao estresse. Arq. Ciênc. Saúde Unipar, Umuarama, v. 18 n. 1, p. 43-49, jan./abr. 2014. Disponível em: <http://www.revistas.unipar.br/index.php/saude/article/download/5157/2983>. Acesso em: 16 dez. 2018.

PAGANINI, T.; FLORES e SILVA, Y. O uso da aromaterapia no combate ao estresse. Arq. Ciênc. Saúde Unipar, Umuarama, v. 18 n. 1, p. 43-49, jan./abr. 2014. Disponível em: <http://www.revistas.unipar.br/index.php/saude/article/download/5157/2983>. Acesso em: 20 dez. 2018.

PEREIRA, M. Recursos técnicos em estética. (Série curso de estética) São Caetano do Sul, SP: Difusão Editora, 2013.

PEREIRA, M. Spaterapia. (Série curso de estética) 1.ed. São Caetano do Sul, SP: Difusão Editora, 2013. 
PEREZ, E.; VASCONCELOS, M. G. Técnicas Estéticas Corporais. 1 ed. São Paulo: Érica, 2014.

SANTOS, Ernestina Xavier dos. Estudo da composição dos monoterpenos das plantas Juniperus Virginiana, Juníperus Bermudiana e Thuja orientalis. 1998.

73 p. monografia (Estudo da composição dos monoterpenos das plantas Juniperus Virginiana, Juníperus Bermudiana e Thuja orientalis)- Faculdade de ciências, Eduardo Mondlane, [S.I.], 1998. Disponível em: <http://hdl.handle.net/123456789/525>. Acesso em: 21 dez. 2018.

SHINAGAWA, FERNANDA BRANCO. Avaliação da composição química de óleos brasileiros de semente de uva (Vitis Vinifera $L$ ) e seu efeito sobre parâmetros bioquímicos e inflamatórios em ratos. Universidade de São Paulo. Tese para orientação do grau de Doutor. São Paulo. 2015. 